\title{
Public health structures attendance during the Ebola outbreak in Guéckédou, Guinea
}

\author{
F. MOISAN ${ }^{1,2}$, A. TRAORE ${ }^{3}$, D. ZOUMANIGUI ${ }^{3}$, J. Y. FEINDOUNO ${ }^{4}$, \\ A. M. SAGNO ${ }^{4}$, T. MOLLET ${ }^{5}$ AND M. BRUYAND ${ }^{1,2 *}$ \\ ${ }^{1}$ French Institute for Public Health Surveillance, Saint-Maurice, France \\ ${ }^{2}$ European Centre for Disease Prevention and Control (ECDC) Deployments in Guinea, WHO/GOARN \\ ${ }^{3}$ Prefectural Health Direction of Guéckédou, Guinea \\ ${ }^{4}$ WHO Guinea \\ ${ }^{5}$ European Centre for Disease Prevention and Control (ECDC), Stockholm, Sweden
}

Received 18 December 2015; Final revision 16 February 2016; Accepted 22 March 2016; first published online 18 April 2016

\section{SUMMARY}

The Ebola virus disease (EVD) outbreak in West Africa may affect healthcare attendance. We describe, in the Guinean prefecture of Guéckédou, trends in attendance of public healthcare structures and the main reported diagnoses over the year following the EVD outbreak notification (March 2014). Monthly numbers of visits and main diagnoses such as malaria, schistosomiasis and measles reported by Guéckédou health centres and health posts were described from January 2012 to March 2015. The median number of visits was 15 724/month. From 1 April to 30 September 2014 (EVD outbreak peak), 90947 visits were reported, representing decreases of $4 \cdot 8 \%$ and $7 \cdot 4 \%$ compared to 2013 and 2012, respectively. Following December 2014 (last EVD notification in Guéckédou), visits increased from 12540 in January to 16032 in March 2015. Malaria seasonality was observed in 2014 with 22519 notifications from 1 April to 31 July. No seasonality was observed for intestinal schistosomiasis (median 485 cases/ month); however, a peak was notified in March 2014 (824 cases). Over the study period, all measles cases were notified in 2015 (183 cases). Reduction in healthcare attendance in Guéckédou was modest during the EVD outbreak. Enhanced infectious disease surveillance is a challenge in this context, due to the impact of EVD on traditional prevention programmes.

Key words: Ebola virus, infectious disease epidemiology, surveillance system.

\section{INTRODUCTION}

In March 2014, the Guinean Ministry of Health issued an alert about an unidentified disease occurring in the forest region of Guinea that turned out to be Ebola virus disease (EVD) [1]. According to the initial epidemiological investigation results, the suspected

\footnotetext{
* Author for correspondence: Dr M. Bruyand, Département des Maladies Infectieuses, Institut de veille Sanitaire, 12 rue du Val d'Osne, 94415 Saint-Maurice Cedex, France.

(Email: m.bruyand@invs.sante.fr)
}

index case had died in December 2013 in Meliandou in Guéckédou prefecture [2]. The first Ebola treatment centre was opened in this prefecture by Médecins sans Frontières in March 2014 [3], this outbreak then spread with a sustained transmission in Guinea, Sierra Leone and Liberia [4]. In October 2015, although the outbreak was still ongoing in Guinea, the last case of EVD diagnosed in Guéckédou was on December 2014 [4].

Regarding other morbid conditions, there is increasing evidence that this EVD outbreak has affected 
the management of infectious diseases in Guinea. From November 2013 to November 2014, a decrease was reported in the numbers of all-cause outpatients visits in the public health facilities in Guinea $(11 \%$ over the period), cases of fever $(15 \%)$, and treatment with antimalarial drugs (oral, 24\%; injectable, 30\%) [5]. HIV prevention programmes were affected as well, from April to December 2014, the proportion of patients receiving antiretroviral therapy who did not present to a scheduled visit at Donka hospital increased from $0 \%$ to $42 \%$, while a decrease in the number of patients in care was evidenced between June and December 2014 [6]. In Macenta, the EVD outbreak also resulted in a major decrease in the utilization of HIV facilities [7]. Regarding other healthcare services, healthcare centre attendance was halved in N'Zérékoré prefecture during the EVD outbreak [8]. Concerning prevention, disruption of childhood vaccination programmes, especially measles vaccination, might result in severe outbreaks in nonimmune populations [9]. In Guinea, the measles vaccination coverage rate preceding the Ebola outbreak was estimated to be under the effectiveness threshold, suggesting that additional breakdown of the routine vaccination programme may lead to severe outbreaks [10]. As a consequence of the EVD outbreak, measles vaccination campaigns were not organized in Guinea in the second half of 2014 [11]. Cholera is circulating in this region, especially in Ghana and Côte d'Ivoire, the latter sharing borders with Liberia and Guinea $[12,13]$. A spread of this outbreak in the countries affected by EVD could have severe consequences [14].

To quantify the impact of the EVD outbreak on the public healthcare system, we describe the attendance at health centres and health posts, and the main diagnoses reported by these structures in the Guinean prefecture of Guéckédou during the year following Ebola outbreak notification.

\section{METHODS}

Data used in this descriptive analysis were provided by the Prefectural Health Direction of Guéckédou. In Guinea, a set of health indicators about public health structures (health centres and health posts) are collected in all prefectures and centralized by the Ministry of Health on a monthly basis through the National System of Health Information and Management (SNIGS) surveillance system. Information is routinely collected, independently of the surveillance specifically implemented in response to the EVD outbreak, through standardized reporting forms in each health centre, including its related health posts. Guéckédou prefecture accounts for 13 health centres and 25 health posts. Data are then computerized at Guéckédou Prefectural Health Direction and communicated to the Ministry of Health. It includes the numbers of visits and diagnosis related to 71 diseases, including 18 with a mandatory notification. Indicators related to maternal and childhood health, prevention of HIV transmission, and financial information about the health structures are also provided [15].

Our study period lasted from January 2012 to March 2015. This period begins 2 years prior the notification of the Ebola outbreak, so as to take into account the influence of seasons on the incidence of a number of infectious diseases such as malaria, which is highly endemic in Guinea [16]. The study ended 3 months after the last EVD case reported by Guéckédou prefecture (December 2014).

Monthly numbers of visits in health centres and health posts reported from January 2012 to March 2015 were provided to describe the trend in attendance at these structures. We used non-parametric methods (Loess regression) to obtain a smooth evolution of the number of visits before and after the first confirmed case of Ebola in Guéckédou (March 2014). The five most frequent diagnoses reported monthly by these public health structures in the surveillance system were also described. This included: malaria, acute respiratory infections, intestinal helminthiasis, vaginal discharge, and gastritis or ulcer. In addition, among infectious diseases requiring a mandatory notification, the six most frequent were described: schistosomiasis, onchocerciasis, bloody diarrhoea, tuberculosis, typhoid fever and measles. Data relating to diseases requiring a mandatory notification were available from January 2012 to December 2014, except for bloody diarrhoea and measles; for these two diseases data collection covered the whole study period.

Information regarding attendance or the number of diagnoses was missing for October 2012, August 2012, and December 2014. In these instances, the mean of the values reported for the preceding and following months was imputed to the missing value.

\section{RESULTS}

\section{Attendance at healthcare structures}

From January 2012 to March 2015, the median number of visits reported each month in Guéckédou prefecture was 15724 [interquartile range (IQR) 14 448-16 930]. 


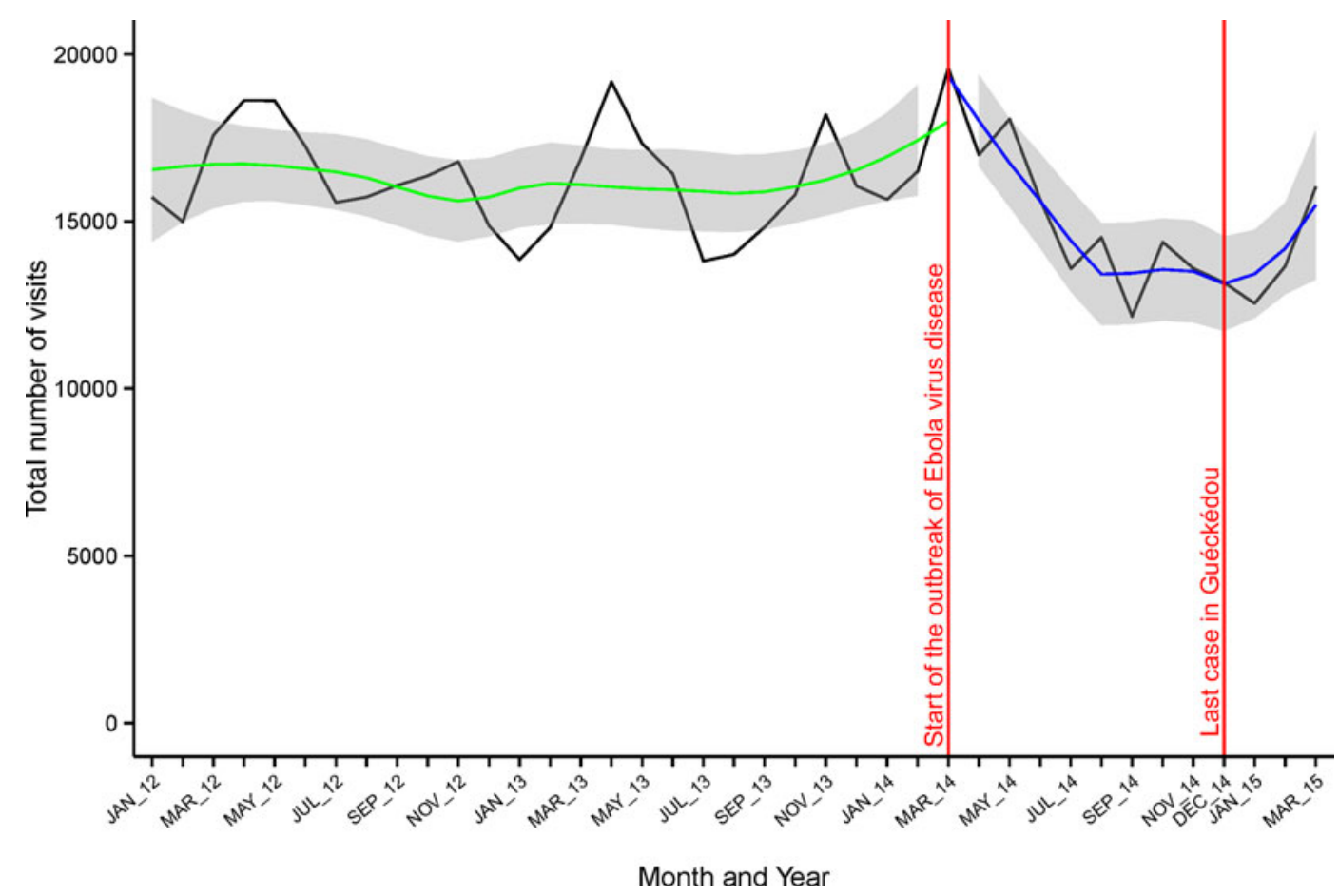

Fig. 1. Attendance at health centres and health posts in the Guinean prefecture of Guéckédou, January 2012-March 2015.

In 2012 and 2013, the season appears to have influenced attendance, with two peaks observed in March-April and October-November in the numbers of visits reported (Fig. 1). Following the Ebola outbreak notification in March 2014, the seasonal peak was evidenced as during the preceding years, and seemed to be in the same range (18 615 visits in May 2012, 19175 visits in April 2013, 19579 visits in March 2014). However, the second peak expected in October-November 2014 was not observed, in contrast the attendance was still falling after July. In 2014, the lowest attendance was observed during September with 12152 visits, compared to 13808 visits in July 2013, which represented the lowest attendance observed in the study period preceding the Ebola outbreak notification.

From April to September, 98225 and 95567 visits were reported in 2012 and 2013, respectively. During the same period in 2014, corresponding to the EVD outbreak peak in Guéckédou, 90947 visits were reported. This represented decreases of $4.8 \%$ and $7 \cdot 4 \%$, respectively, compared to 2013 and 2012.

Of note, following the last EVD notification in Guéckédou, the number of visits increased between January and March 2015 (from 12540 to 16032 visits). This was lower than in March 2014 (19 579 visits), but was in the same range as in March 2012 (17 578 visits) and 2013 (16 870 visits).

\section{Trends of the main diagnoses}

Of 591751 diagnoses reported during the study period, malaria accounted for $214155(36 \%)$, acute respiratory infections for $127022(21 \%)$, intestinal helminthiasis for $69744(12 \%)$, vaginal discharge for $26946(5 \%)$, and gastritis or ulcer for $18481(3 \%)$.

Regarding malaria, the monthly median number of diagnoses reported over the study period was 5370 (IQR 4821-6052). The expected seasonality was observed during the study period, including the EVD outbreak, with 27890,25827 and 22519 malaria cases reported from April to July in 2012, 2013 and 2014, respectively (Fig. 2).

Concerning acute respiratory infections, a higher number of diagnoses was noted during the periods of April-June (median: 3500 diagnoses/month) and September-December (median: 3300 diagnoses/ month) in 2012 and 2013, respectively. However, in 2014, for the latter period, fewer diagnoses were recorded (median: 2837 diagnoses/month; Fig. 2).

We did not observe major changes in the other diagnoses (helminthiasis, vaginal discharge, ulcer) reported 


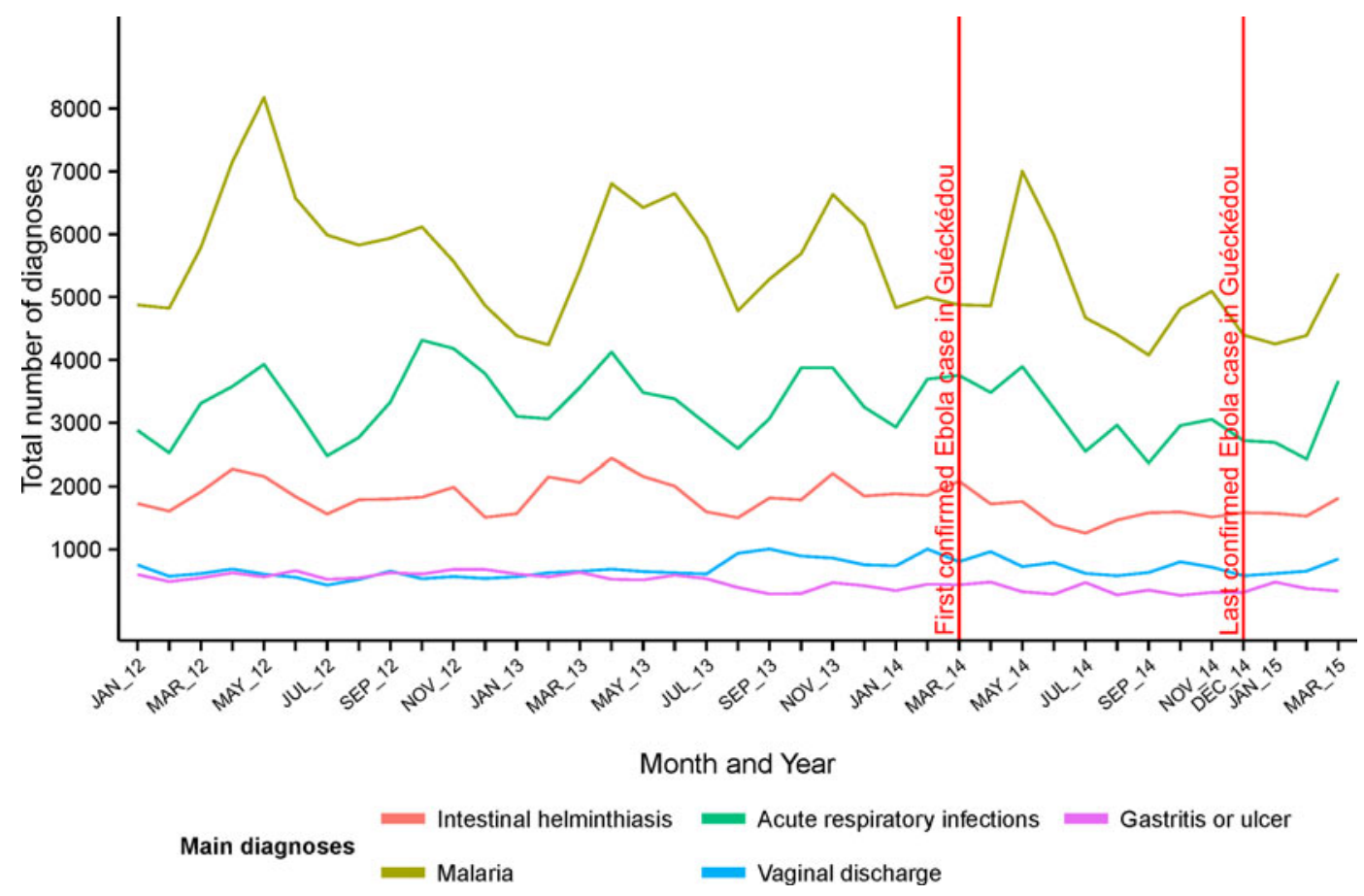

Fig. 2. Main diagnoses reported by health centres and health posts in the Guinean prefecture of Guéckédou, January 2012-March 2015.

between March and December 2014 compared to 2012 and 2013 for (Fig. 2).

\section{Evolution of the number of notifiable diseases}

Intestinal schistosomiasis was the most frequent mandatory notifiable disease, a median total of 485 cases were reported monthly (IQR 299-589). Seasonality was not observed during the study period, but the number of cases seemed to decrease after June 2013, and an unexpected peak was observed in March 2014 (824 cases). In 2013 and 2012 the highest values were observed in April with 604 and 662 cases, respectively (Fig. 3). The number of urinary schistosomiasis diagnoses reported monthly was much lower (median: five cases) but a peak was observed between April and August 2014 with in median of 52 cases per month and a peak of 89 cases in June.

Over the study period, a median of 42 bloody diarrhoea diagnoses were reported monthly (IQR 28-67). Peaks were observed each year in March (Fig. 4). Following March 2014, the number of bloody diarrhoea cases declined to $<30$ cases/month [a median number of 20 cases were reported monthly from April 2014 to March 2015 (IQR 12-27)]. From January to March 2014, a total of 261 bloody diarrhoea cases was reported in Guéckédou, 152 (58\%) of these being notified by Guendembou and Tékoulo sub-prefectures; both sub-prefectures had notified probable and confirmed EVD cases in the first months of the outbreak. In the preceding year from January to March 2013, of 178 cases of bloody diarrhoea notified in Guéckédou, $75(42 \%)$ were notified from these sub-prefectures.

No measles cases were notified between April 2012 and December 2014 (Fig. 4). A total of 183 cases was notified during the first trimester of 2015 in Guéckédou (January: 45 cases; February: 45 cases, March: 93 cases), confirming the measles outbreak reported in N'Zérékoré prefecture at that time [11].

Finally, the number of onchocerciasis diagnoses reported has markedly reduced during the Ebola outbreak, while no important trend was observed regarding typhoid fever and tuberculosis (Fig. 4).

\section{DISCUSSION}

This study shows that, in Guéckédou prefecture, attendance in non-hospital public health structures decreased by $5-7 \%$ during the first 6 months of the EVD outbreak, compared to the same period in the preceding years.

This reduction might be considered as modest; however, important changes were observed regarding 


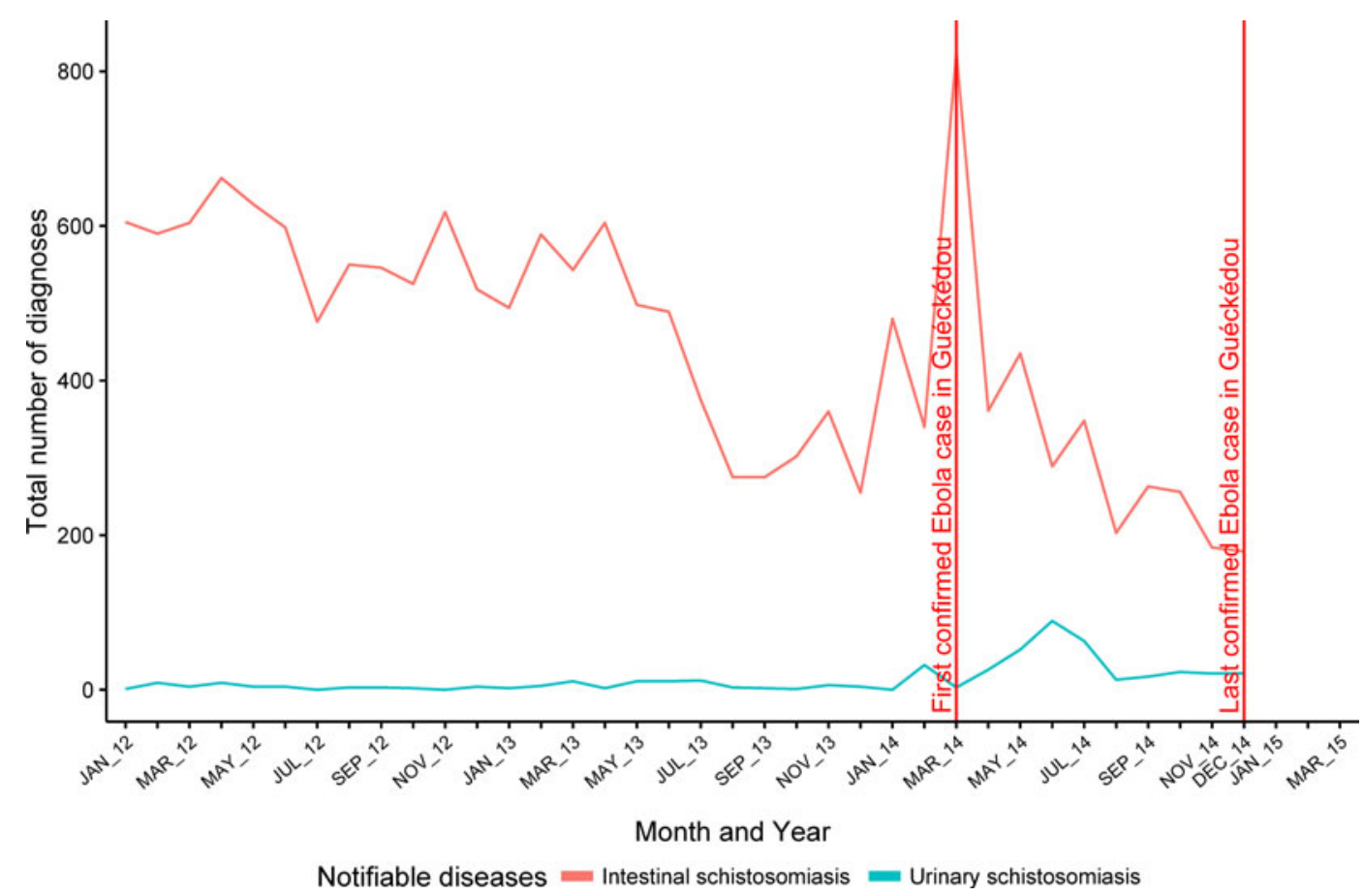

Fig. 3. Schistosomiasis reported by health centres and health posts in the Guinean prefecture of Guéckédou, January 2012-March 2015.

specific diagnoses (bloody diarrhoea, schistosomiasis), while for some high-incidence diseases, such as malaria, no major changes were observed.

During the EVD outbreak in Guéckédou, no health centres or health posts were closed due to medical staff contamination by Ebola virus. Thus, the population had access to all health structures over the study period in Guéckédou, especially during the EVD outbreak.

In N'Zérékoré prefecture, healthcare attendance decreased by around half during the EVD outbreak,

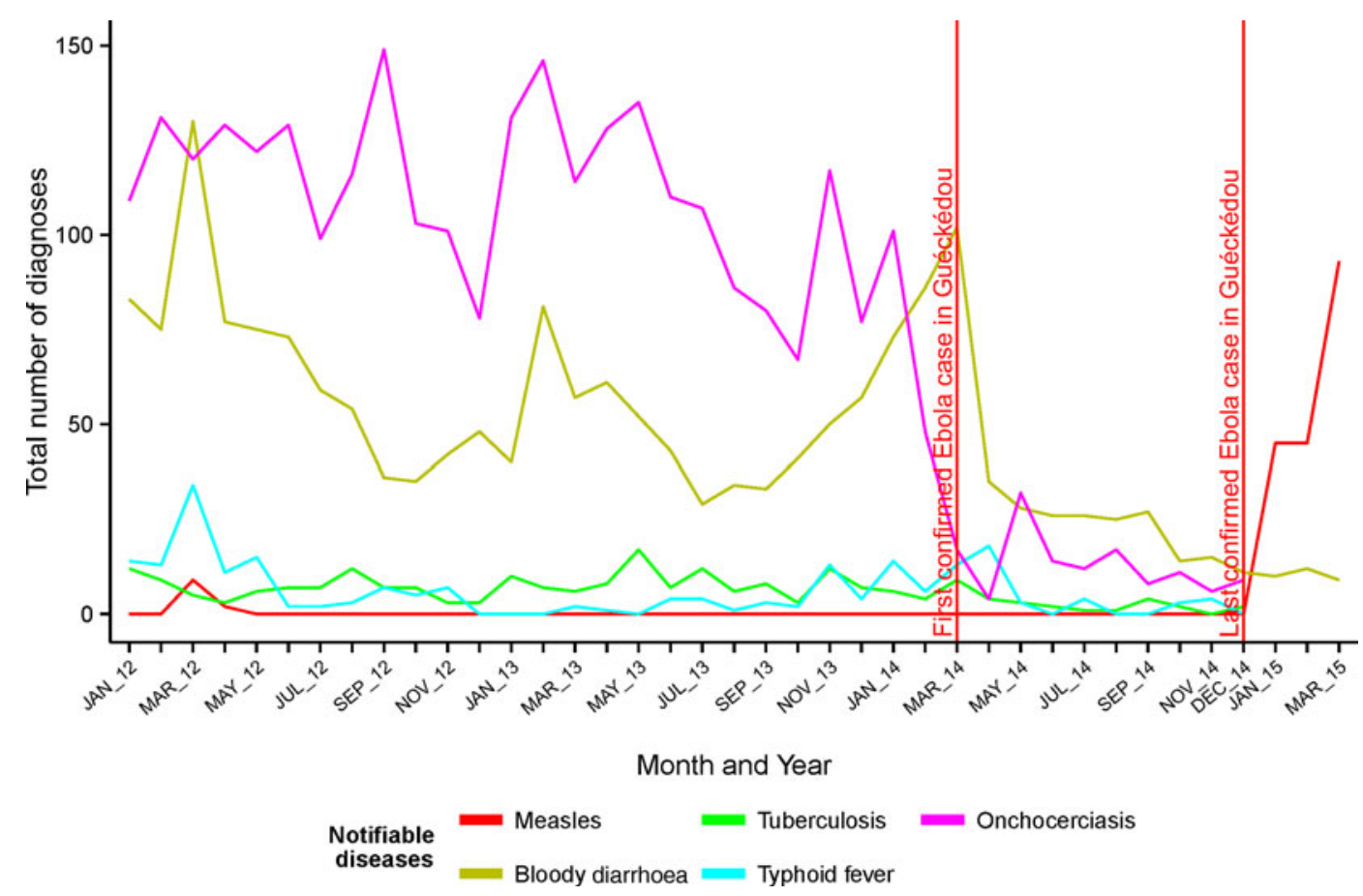

Fig. 4. Notifiable diseases reported by health centres and health posts in the Guinean prefecture of Guéckédou, January 2012-March 2015. 
which occurred there between September 2014 and February 2015 [8]. Thus, in Guéckédou no major changes were observed in healthcare attendance while in N'Zérékoré, another prefecture located in the Guinean forest region, healthcare attendance was halved during the EVD outbreak. A cross-sectional survey performed in Guinean health facilities has evidenced that decline in healthcare attendance might be driven by the population's fear [5]. We can speculate that in N'Zérékoré the population's fear has preceded the occurrence of the EVD outbreak, possibly worsening the fall in healthcare attendance. In Guéckédou, the epicentre of the outbreak, the population's fear might have occurred at the same time as the outbreak, perhaps leading to a lower decrease in healthcare attendance. However, we were not able to fully describe the social characteristics of these prefectures to completely understand this difference.

In a study assessing the effect of the EVD outbreak on malaria case management in Guinea, a cross-sectional survey of health facilities was conducted in eight Guinean prefectures (the four most affected by the EVD outbreak, and four without any reported EVD cases). The most common explanation provided by healthcare workers for the decrease in healthcare attendance were: patient's fear of healthcare workers, contracting EVD during a visit to a health facility, and being sent to an Ebola treatment centre. These results suggest that the EVD outbreak has affected trust in the public health system and led to the population's fear in accessing it [5].

We noted an increase in reported bloody diarrhoea and intestinal schistosomiasis in the weeks preceding the EVD outbreak notification, especially in Tékoulo and Guendembou sub-prefectures; two sub-prefectures where EVD cases were diagnosed at the beginning of the outbreak. Reported schistosomiasis diagnoses are clinically based, as biological confirmation is not routinely available, and diarrhoea is a common symptom of this disease. However, it is of note that in all the diseases reported, those for which a peak was observed in the weeks preceding the EVD outbreak notification share common symptoms with EVD: diarrhoea and bloody diarrhoea, even if bleeding is not among the most frequent symptoms reported during the current EVD outbreak [17, 18]. Except for malaria, biological confirmation is not routinely available for the diagnoses reported here, and lethality rates are not reported. Thus, we were not able to further describe the aetiology of these peaks of diarrhoea diagnoses.

The absence of measles diagnosis reported during a 32-month period strongly suggests an underreporting of this disease in Guéckédou, as of 28 March 2014 the measles outbreak notified in January 2014 in Guinea has resulted in 4689 cases and 11 deaths [19]. However, despite this limitation, this surveillance system detected the measles outbreak occurring in 2015. The reasons of this underreporting are unclear and should be further explored, as measles diagnosis is clinically based on the occurrence of typical symptoms.

Surveillance systems in Guinea need to be implemented according to international standards in a resilient health system able to respond both to routine and unexpected challenges [20]. However, it should be acknowledged that data collected through current routine surveillance in Guinea were available to perform this descriptive analysis despite the limitations of these systems. This suggests that the implementation of an efficient surveillance system in Guinea might rely on existing structures.

This study also presents a number of limitations. Rapid diagnosis and malaria treatment are free and widely available in public health structures in Guinea; however, the other diagnoses reported by these health structures are mainly clinically based, even for infectious diseases. Thus, misdiagnosis issues cannot be excluded and the influence of this potential bias could not be assessed. Data are collected on a monthly basis, leading to a poor reactivity of this system, and numerous contributors are involved (health posts, health centres, Guéckédou Prefectural Health Direction, and Ministry of Health). This can lead to potential mistakes in this system, or a lack of reactivity.

This study does not include data related to care provided in hospitals and the private sector, especially traditional healers. The lack of hospital-based information is not expected to be a major issue, as hospital attendance may be quite low compared to all other public health structures. In contrast, the overall health system attendance is underestimated due to the absence of information regarding traditional healers' activity, and information bias cannot be excluded if patients with specific conditions were more likely to visit to traditional healers. In Guinea, prior to the EVD outbreak, it was estimated that $57 \%$ of children presenting febrile illness were treated in the public sector, and 13\% by traditional healers, which accounts for the most frequent alternative to public sector [16]. This highlights the important role of traditional healers in Guinea.

To fully understand the evolution of healthcare attendance during the EVD outbreak in each prefecture, a detailed social structure should be considered, including health system acceptance. 
Observed numbers of patients attending healthcare centres and numbers of specific diagnoses were reported rather than incidence estimates. This is due to the lack of reliable estimations of the Guéckédou population size.

\section{CONCLUSION}

Active transmission of Ebola virus was still ongoing in Guinea in October 2015, at that time the last case in Guéckédou was in December 2014. Data observed in this context appear to show that lower attendance at public health structures observed during the EVD outbreak was not persistent in this Guinean prefecture. The emergence of measles recalls the marked consequences of an EVD outbreak, related to the higher risk of occurrence of other infectious diseases outbreaks. This highlights the necessity of implementing efficient surveillance and outbreak response in resilient health systems, in implementing these measures social specificities should be considered.

\section{ACKNOWLEDGMENTS}

The authors acknowledge Dr Henriette De Valk (French Institute for Public Health Surveillance) and Dr Josep Jansa (European Centre for Disease Prevention and Control) for their input in this manuscript.

\section{DECLARATION OF INTEREST}

None.

\section{REFERENCES}

1. WHO. One year into the Ebola Epidemic, a deadly, tenacious and unforgiving virus (http://www.who.int/csr/ disease/ebola/one-year-report/ebola-report-1-year.pdf). Accessed 15 December 2015.

2. Baize $\mathbf{S}$, et al. Emergence of Zaire Ebola virus disease in Guinea. New England Journal of Medicine 2014; 371: 1418-1425.

3. Médecin sans frontières. Pushed beyond our limits. One year of struggle against the worst Ebola outbreak ever reported [in French] (http://www.msf.lu/fileadmin/ WEBLibrary/1_Sur_le_terrain/Autres_maladies/MSF_ EbolaReport_FR.pdf). Accessed 15 December 2015.

4. WHO. Ebola Situation Report (http://apps.who.int/ ebola/current-situation/ebola-situation-report-21-october2015). Accessed 15 December 2015.

5. Plucinski MM, et al. Effect of the Ebola-virus-disease epidemic on malaria case management in Guinea,
2014: a cross-sectional survey of health facilities. Lancet Infectious diseases 2015; 15: 1017-1023.

6. Ndawinz JD, et al. Prevention of HIV spread during the Ebola outbreak in Guinea. Lancet 2015; 385: 1393.

7. Leuenberger D, et al. Impact of the Ebola epidemic on general and HIV care in Macenta, Forest Guinea, 2014. AIDS 2015; 29: 1883-1887.

8. Perron E, et al. Reduction in attendance of health care centres, during Ebola outbreak in N'Zérékoré prefecture, Guinea, November 2013-March 2015. European Scientific Conference on Applied Infectious Disease Epidemiology. Stockholm, Sweden, 2015.

9. Takahashi S, et al. Reduced vaccination and the risk of measles and other childhood infections post - Ebola. Science 2015; 347: 1240-1242.

10. Doumtsop JG, et al. An evaluation of the 2012 measles mass vaccination campaign in Guinea. Pan African Medical Journal 2014; 17: 4.

11. UNICEF. Guinea, measle situation report (http://www. unicef.org/appeals/files/UNICEF_Guinea_Measles_Sit Rep_24Mar2015.pdf). Accessed 15 December 2015.

12. Ministère de la santé de Côte d'Ivoire. Press conference: cholera outbreak in Côte d'Ivoire [in French] (http:// www.sante.gouv.ci/index2.php?page $=$ actu\&ID=231). Accessed 15 December 2015.

13. UNICEF. Cholera update in the West and Central Africa Region (regional update, 2014, week 49) (http:// www.unicef.org/cholera/files/Cholera_regional_update_ W49_2014_West_and_Central_Africa.pdf). Accessed 15 December 2015.

14. Azman AS, et al. Outbreaks of cholera in the time of Ebola: pre-emptive action needed. Lancet 2015; 385: 851.

15. WHO. WHO Regional office of Africa, Guinea profile $\quad($ http://www.google.fr/url?sa $=\mathrm{t} \& \mathrm{rct}=\mathrm{j} \& \mathrm{q}=\& \mathrm{esrc}=$ s\&source $=$ web\&cd $=3 \&$ ved $=0 \mathrm{CC}$ QFjAC\&url $=\mathrm{http}: / / \%$ $3 \mathrm{~A} \% 2 \mathrm{~F} \% 2 \mathrm{Fwww}$.afro.who.int $\% 2$ Findex.php\%3Foption $\% 3$ Dcom_docman $\% 26$ task $\% 3$ Ddoc_download $\% 26$ gid $\%$ 3D4262\&ei=kK-SVbSqIcjt-QG2zoD4DQ\&usg=AFQj CNG3oX6A88L_RIFqx5dAm1gqPv8oMQ\&bvm=bv. 96783405,d.ZGU). Accessed 15 December 2015.

16. INS, MSHP, ICFI. Guinea. Demographic and health survey producing multiple information [in French]. Calverton, MD: Institut National de la Statistique, Ministère de la Santé et de l'Hygiène Publique, ICF International, 2012 (http://www.dhsprogram.com/pubs/ pdf/FR280/FR280.pdf). Accessed 15 December 2015.

17. Bah EI, et al. Clinical presentation of patients with Ebola virus disease in Conakry, Guinea. New England Journal of Medicine 2015; 372: 40-47.

18. Barry M, et al. Ebola outbreak in Conakry, Guinea: epidemiological, clinical, and outcome features. Medecine et maladies infectieuses 2014; 44: 491-494.

19. UNICEF. Guinea CO Humanitarian Situation Report (http://www.unicef.org/appeals/files/UNICEF_Guinea_ SitRep_29_MAR_2014.pdf). Accessed 15 December 2015.

20. Kieny MP, Dovlo D. Beyond Ebola: a new agenda for resilient health systems. Lancet 2015; 385: 91-92. 